\title{
Interprofessional Collaborative Practice in the Medical Intensive Care Unit: a Survey of Caregivers' Perspectives
}

\author{
Debbie W. Chen, $M D^{7}$, Angela M. Gerolamo, PhD, RN², Elissa Harmon, RN, DNP, CCRN, PHNA-BC 3 , \\ Anna Bistline, $B S^{4}$, Shoshana Sicks, EdM ${ }^{5}$, and Lauren Collins, $M D^{5,6}$ \\ 'Department of Internal Medicine, Thomas Jefferson University Hospital, Philadelphia, PA, USA; ${ }^{2}$ Jefferson College of Nursing, Philadelphia, PA, USA; \\ ${ }^{3}$ Department of Nursing, Thomas Jefferson University Hospital, Philadelphia, PA, USA; ${ }^{4}$ Sidney Kimmel Medical College, Philadelphia, PA, USA; \\ ${ }^{5}$ Jefferson Center for Interprofessional Practice and Education, Philadelphia, PA, USA; ${ }^{6}$ Department of Family and Community Medicine, Sidney \\ Kimmel Medical College, Thomas Jefferson University, Philadelphia, PA, USA.
}

\begin{abstract}
BACKGROUND: Research on caregivers, defined as designated family members or support persons, in the medical intensive care unit (MICU) has primarily focused on their emotional needs and experiences, thus leaving a gap in knowledge related to their perceptions of team dynamics.
\end{abstract}

OBJECTIVE: To examine caregivers' perceptions of team interactions and competencies for interprofessional collaborative practice (IPCP) and overall satisfaction with the MICU team.

METHODS: The Support Person Jefferson Teamwork Observation Guide (JTOG) ${ }^{\mathrm{TM}}$ was administered to a convenience sample of caregivers in the MICU at a large urban academic medical center between May 2016 and December 2016.

RESULTS: One hundred sixty-one JTOG surveys were completed. Caregivers agreed on the importance of healthcare professionals working together as a team to provide patient care (3.97 out of 4.0 on Likert response scale where 1 is "Not at all important" and 4 is "Extremely important") and were satisfied with the MICU team (3.74 out of 4.0), positively evaluating the four core competencies for IPCP (3.55 for values/ethics, 3.58 for interprofessional communication, 3.61 for roles/responsibilities, and 3.64 for teams/teamwork) and the patient/familycenteredness sub-competency (3.58 out of 4.0). There was a strong positive correlation between caregivers' Global JTOG scores and overall satisfaction with the MICU team ( $r=0.596, p<0.01)$. Caregivers' comments about factors that affected their experience focused on aspects of interprofessional communication and patient/family-centeredness.

CONCLUSION: Findings underscore the importance of interprofessional communication and providing patient/ family-centered care. Assessing caregivers' perceptions of IPCP can provide a critical lens into team functioning and, thus, be used to identify teams' strengths as well as opportunities for improvement.

Electronic supplementary material The online version of this article (https://doi.org/10.1007/s11606-018-4623-3) contains supplementary material, which is available to authorized users.

Received December 12, 2017

Revised May 15, 2018

Accepted July 18, 2018

Published online August 15, 2018
KEY WORDS: critical care; evaluation; communication; clinical skills assessment; teamwork; collaborative practice; interprofessional education; caregivers' perceptions.

$\mathrm{J}$ Gen Intern Med 33(10): 1708-13

DOI: $10.1007 / \mathrm{s} 11606-018-4623-3$

(C) Society of General Internal Medicine 2018

\section{BACKGROUND}

In its landmark publication, "Crossing the Quality Chasm," the Institute of Medicine (IOM) named patient-centered care as one of the six specific aims for improving the quality of healthcare. ${ }^{1}$ This publication sparked a renewed interest in interprofessional education (IPE), which is essential to prepare future providers for team-based collaborative practice (CP) with the goal of enhancing patient experience, improving health outcomes, and decreasing healthcare costs. ${ }^{2-6}$ In response to the IOM's 2001 publication, clinical practice guidelines to promote involvement of family members in the intensive care unit were published. ${ }^{7}$ Among the recommendations were early and repeated care conferencing, family support surrounding a patient's death, and inclusion of family during daily rounds and resuscitation. Effective collaboration is particularly relevant in the medical intensive care unit (MICU) setting where the most critically ill patients are cared for by interprofessional teams in a high-acuity, high-stress environment. The MICU team includes providers from multiple professions and subspecialties who work together to balance medical decisions that impact patient outcomes and quality of life against cost given the scarcity of resources.

In the MICU setting, caregivers (defined as designated family members or support persons) frequently interact with the healthcare team and influence the delivery of medical therapies and interventions. Research on caregivers in the MICU setting has focused primarily on their emotional needs and experiences, thus leaving a gap in knowledge related to their perceptions of team dynamics and healthcare delivery. ${ }^{8-}$ ${ }^{10}$ Given the acuity of patients in the MICU and the role of caregivers as advocates in this setting, it is necessary to elicit caregivers' perceptions of the behaviors of interprofessional teams in the MICU. 


\section{OBJECTIVE}

The aim of this study is to examine caregivers' perceptions of team interactions and competencies for interprofessional collaborative practice (IPCP) and overall satisfaction with care provided by the MICU team.

\section{METHODS}

Study Design. This study used a descriptive design. The Support Person Jefferson Teamwork Observation Guide $(\mathrm{JTOG})^{\mathrm{TM}}$ was administered via secure mobile tablet computers by research assistants who were trained prelicensure students not part of the healthcare team. Caregivers were consented for the study and informed that data collected would be used for research purposes. All members of the MICU team were blinded and had no knowledge of which caregivers were participating in the study. Data was collected between May 2016 (beginning of the Summer semester for prelicensure students) and December 2016 (end of the Fall semester for prelicensure students). Due to availability of the research assistants, data collection in the MICU was conducted for a total of $147 \mathrm{~h}$ over the 8-month study period (range from 8 to $26.3 \mathrm{~h}$ per month). Research assistants collected data for 2 to $3 \mathrm{~h}$ per shift, which were scheduled between 10:30 AM and 6:30 PM 7 days per week. No data was collected about the individual patients whose caregivers participated in the study. This study was exempt from Institutional Review Board review.

Setting. The study was conducted at a 937-bed urban tertiary care academic medical center with a 25 -five bed MICU that manages complex critically ill patients. Between May 2016 and December 2016, a total of 931 patients were cared for in the MICU. The top three diagnoses among MICU admissions in 2016 were as follows: (1) severe sepsis and septic shock; (2) respiratory failure; and (3) gastrointestinal bleed.

The MICU at our institution consists of two separate units with a "closed format" setting, but different staffing coverage. Seventeen beds are managed in the resident- and fellowstaffed MICU, and the remaining eight beds are managed in the nurse practitioner-staffed MICU. In the resident- and fellow-staffed MICU, the general workflow at the time of study was as follows: in the morning, an interprofessional team of charge nurse, staff nurses, respiratory therapist, physical and occupational therapists, and wound care nurses met for brief Awakening and Breathing Coordination, Delirium monitoring/management and Early exercise/mobility (ABCDE) bundle rounds. This activity was followed by multidisciplinary bedside team rounds, which was led by the critical care attending physician and consisted of residents, a pulmonary/critical care fellow, staff nurses, pharmacists, patients (if able to participate), and families/support persons (if present at the bedside). In the afternoon, workflow was focused on the completion of tasks related to patient care, discussion about medical management with medical and surgical subspecialty teams that were consulted, and reassessment of patients. Healthcare professionals on the consulted subspecialty teams evaluated patients throughout the day. In the late afternoon, the attending, residents, and fellows met again to discuss interval events and consultants' recommendations. In the evening, patients were signed out to the overnight team of residents and one fellow. In the nurse practitioner-staffed MICU, workflow was similar except that three nurse practitioners took the place of residents and fellows during the day, there was a different critical care attending physician, dieticians and case managers sometimes attended the morning interprofessional bedside team rounds, and evening coverage was provided by a hospitalist.

Instrument. The original JTOG, now known as the Team JTOG, was initially validated in 2016 to assist prelicensure students in understanding the characteristics of wellfunctioning teams through the observation of teams in action. ${ }^{11}$ The Team JTOG consists of 17 items (14 quantitative and three qualitative questions). The 14 quantitative questions use the Likert response scale of 1 (strongly disagree) to 4 (strongly agree) and are each mapped to the 2011 Interprofessional Education Collaborative (IPEC) core competencies for IPCP: values/ethics, interprofessional communication, roles/ responsibilities, and teams/teamwork (Table 1). ${ }^{12,13}$ One additional domain, leadership, was included based on a review of the literature pertaining to effective teamwork.

An Interprofessional Education Research and Evaluation committee at our institution designed and refined three additional versions of the JTOG (Patient, Support Person, and Individual). Each of these three versions contain items similar to those in the Team JTOG, but the wording of individual items was amended to reflect the user's role (e.g. learner, provider, patient or family member) and the unit being

Table 1 Definition of the Four IPEC Core Competencies for Interprofessional Collaborative Practice (IPCP), and the Patient/ Family-Centeredness Sub-competency

\begin{tabular}{ll}
\hline \hline $\begin{array}{l}\text { IPEC core competencies } \\
\text { for IPCP }\end{array}$ & Definition \\
\hline Values/ethics & $\begin{array}{l}\text { Professionalism, shared virtues, trust, } \\
\text { and mutual respect }\end{array}$ \\
Interprofessional & $\begin{array}{l}\text { Effective information exchange; being } \\
\text { available in place, time, and } \\
\text { communication }\end{array}$ \\
$\begin{array}{l}\text { knowledge; engaging in active } \\
\text { listening; and conveying openness } \\
\text { Roles/responsibilities }\end{array}$ & $\begin{array}{l}\text { Expertise in one's own profession, } \\
\text { recognizing one's limitations, and } \\
\text { understanding others' roles in relation } \\
\text { to oneself } \\
\text { Cooperating in patient-centered care } \\
\text { delivery, and collaborating with others } \\
\text { through shared problem-solving and } \\
\text { shared decision-making } \\
\text { Respect and responsiveness to patients' } \\
\text { and families' needs and values }\end{array}$ \\
&
\end{tabular}


measured (e.g. a care team versus an individual on the team). As in the Team JTOG, each question maps directly to one of the IPEC core competencies for IPCP.

The Support Person JTOG, which was designed to elicit the perspective of family members and/or support persons regarding team functioning, was adapted directly from the recently validated Patient JTOG. ${ }^{14}$ The Support Person JTOG consists of ten items (eight quantitative and two qualitative questions) with four quantitative questions mapped directly to one of the 2011 IPEC core competencies for IPCP and two quantitative questions focused on patient/family-centeredness, a subcompetency under values/ethics (Table 2, see the Supplementary Appendix). These six quantitative questions use the Likert response scale of 1 (strongly disagree) to 4 (strongly agree) with a "Not observed" option available. The remaining two quantitative questions ask about overall satisfaction with the team and general attitude regarding the importance of interprofessional team-based care.

Population. The surveys were administered to a convenience sample of caregivers in both the resident- and fellow-staffed MICU and in the nurse practitioner-staffed MICU. Caregivers were selected at random based on availability and presence at the patients' bedside, regardless of patients' length of stay in the MICU. The exclusion criteria were age less than 18, and caregivers who did not speak English. Caregivers were asked to answer the survey questions based on their real-time observations of and interactions with the care team in the MICU on the day that the survey was administered. Caregivers were only surveyed once during the study.

Analysis. Respondents with no more than two missing or "Not observed" responses for the quantitative questions were included in analyses. All quantitative analyses were conducted using IBM SPSS Statistics for Windows, version 22 (IBM Corp., Armonk, NY, USA). Descriptive statistical analysis was performed for all categorical variables. The

Table 2 Questions on the Support Person JTOG That Are Mapped to One of the Four IPEC Core Competencies for IPCP or Focused on the Patient/Family-Centeredness Sub-competency

\begin{tabular}{ll}
\hline \hline Support Person JTOG Question & Competency addressed \\
\hline $\begin{array}{l}\text { Team members engaged in friendly } \\
\text { interaction with one another }\end{array}$ & Teamwork \\
$\begin{array}{l}\text { Each member of the team seemed } \\
\text { prepared to discuss the current health } \\
\text { of the patient using his/her professional } \\
\text { knowledge }\end{array}$ & Roles/responsibilities \\
$\begin{array}{l}\text { Team member appeared to listen to one } \\
\text { another }\end{array}$ & Communication \\
$\begin{array}{l}\text { Each member of the team appeared to } \\
\text { value the opinions of other members }\end{array}$ & Values/ethics \\
$\begin{array}{l}\text { Each member of the team made me } \\
\text { feel as though I was an important } \\
\text { member of the team }\end{array}$ & $\begin{array}{l}\text { Patient/family-centeredness } \\
\text { (sub-competency) }\end{array}$ \\
$\begin{array}{l}\text { Each member of the team seemed to } \\
\text { respect the patient's wishes about his/ } \\
\text { her care }\end{array}$ & $\begin{array}{l}\text { Patient/family-centeredness } \\
\text { (sub-competency) }\end{array}$ \\
\hline
\end{tabular}

average values for caregivers' assessment of competency in IPCP were calculated based on their responses to the six quantitative questions that were mapped to the four IPEC core competencies and the patient/family-centeredness subcompetency. The sum of these six quantitative questions comprise the Global JTOG score (possible range from 6 to 24). Pearson's correlation was used to assess the relationship between caregivers' Global JTOG scores and single-item overall satisfaction with the MICU team. The open-ended responses were mapped to the four IPEC core competencies for IPCP and the patient/family-centeredness sub-competency. Some responses mapped to more than one competency. Summary statistics were then used to identify the frequency that the caregivers' comments addressed these competencies and sub-competency.

\section{RESULTS}

Demographics. One hundred sixty-one Support Person JTOG surveys were completed by caregivers between May 2016 and December 2016. Seventy percent of respondents were female with a Caucasian predominance $(n=121,75 \%)$. Sixty-three percent were aged 50 years or older (Table 3 ).

JTOG Results. Six caregivers were excluded for missing responses $(3.7 \%)$. Of these, five were missing three items each, and the sixth was missing four. After excluding these six respondents, there were a total of 34 missing responses in the entire dataset (2.7\% of all data). Caregivers agreed on the importance of healthcare professionals working together as a team in providing patient care (3.97 out of 4.0 on Likert response scale) and were generally satisfied with the MICU team (3.74 out of 4.0), positively evaluating the four IPEC core competencies for IPCP and the patient/familycenteredness sub-competency (Fig. 1). The average Global JTOG score was 21.5 out of 24 . There was a strong positive correlation between caregivers' Global JTOG scores and the single-item question on overall satisfaction with the MICU team (Pearson's correlation coefficient, $r=0.596, p<0.01$ ) (Fig. 2). Multiple team members were identified by the respondents as being involved in the overall care of the patient,

Table 3 Participant Demographics. Demographic Data on Caregivers Who Participated in the Study

\begin{tabular}{ll}
\hline \hline Characteristics & Overall $(\boldsymbol{n}=\mathbf{1 5 5})$ \\
\hline Age $\geq 50$ years old & $63 \%(101)$ \\
Female & $70 \%(113)$ \\
Hispanic or Latino origin & $3 \%(5)$ \\
Ethnicity & \\
Caucasian & $75 \%(121)$ \\
African American & $14 \%(22)$ \\
Asian or Pacific Islander & $5 \%(8)$ \\
Multi-racial & $3 \%(5)$ \\
\hline
\end{tabular}




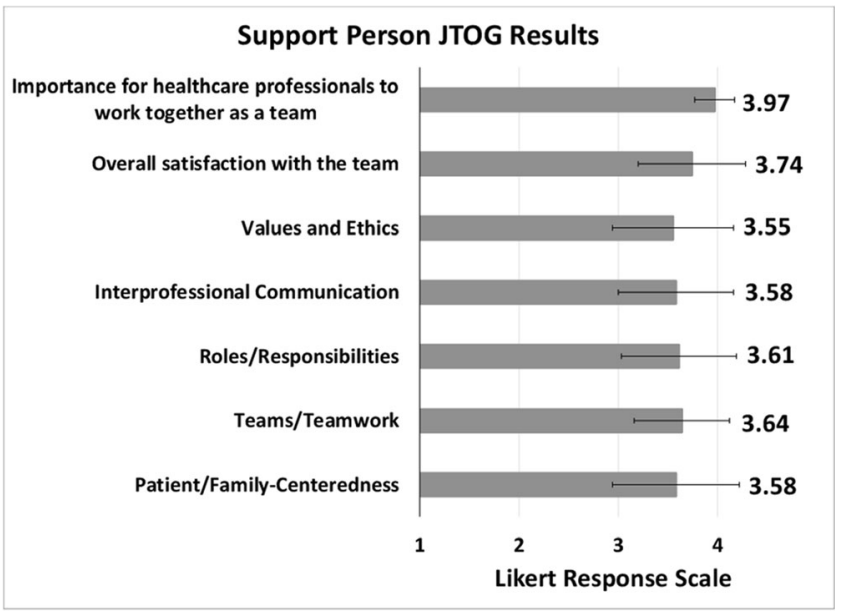

Figure 1 Support Person JTOG results. Caregivers agreed on the importance of healthcare professionals working together as a team in providing patient care $(3.97 \pm 0.20$ out of 4.0 where 1 is "Not at all important" and 4 is "Extremely important"). Overall satisfaction with the MICU team was $3.74 \pm 0.54$ out of 4.0 where 1 is "Not at all satisfied" and 4 is "Extremely satisfied." The average values of caregivers' assessments of each of the four IPEC core competencies for IPCP were as follows: $3.55 \pm 0.61$ for values/ethics, $3.58 \pm 0.58$ for interprofessional communication, $3.61 \pm \mathbf{0 . 5 8}$ for roles/responsibili-

ties, and $3.64 \pm 0.48$ for teams/teamwork where 1 is "strongly

disagree" and 4 is "strongly agree." For the patient/familycenteredness sub-competency, the MICU team scored an average of $3.58 \pm 0.64$.

including nurses and nurse practitioners (96\%), physicians $(92 \%)$, respiratory therapists $(44 \%)$, x-ray technologists (40\%), physical therapists (29\%), case managers (24\%), and pastoral care $(20 \%)$.
Table 4 Factors That Positively and Negatively Affected Caregivers' Experience in the MICU. Caregivers' Answers Referenced Key Characteristics of All Four IPEC Competencies for IPCP (Values/ Ethics, Interprofessional Communication, Roles/Responsibilities, and Teams/Teamwork) as well as the Patient/Family-Centeredness Sub-competency

\begin{tabular}{|c|c|c|}
\hline & $\begin{array}{l}\text { Positive } \\
\text { factors }\end{array}$ & $\begin{array}{l}\text { Negative } \\
\text { factors }\end{array}$ \\
\hline Responses $(n, \%)$ & $140(90 \%)$ & $113(73 \%)$ \\
\hline Competencies for IPCP & & \\
\hline Values/ethics & $7(5 \%)$ & $0(0 \%)$ \\
\hline $\begin{array}{l}\text { Interprofessional } \\
\text { communication }\end{array}$ & $71(51 \%)$ & $14(12 \%)$ \\
\hline Roles/responsibilities & $31(22 \%)$ & $7(6 \%)$ \\
\hline Teams/teamwork & $18(13 \%)$ & $9(8 \%)$ \\
\hline Sub-competency & & \\
\hline Patient/family-centeredness & $35(25 \%)$ & $17(15 \%)$ \\
\hline
\end{tabular}

When asked via the open-ended questions about factors that positively and negatively affected their experience as a caregiver, $90 \%$ and $73 \%$ responded, respectively. In the latter group, $64 \%$ of respondents reported that they did not have a negative experience in the MICU. Caregivers' answers referenced key characteristics of all four IPEC competencies for IPCP as well as the patient/family-centeredness subcompetency (Table 4). ${ }^{12}$ Caregivers' comments about positive and negative factors influencing their observations of and interactions in the MICU primarily focused on aspects related to interprofessional communication and patient/family-centeredness. Caregivers expressed their desire to be included in patient care decisions and to ensure that their loved one is being treated with respect and dignity.

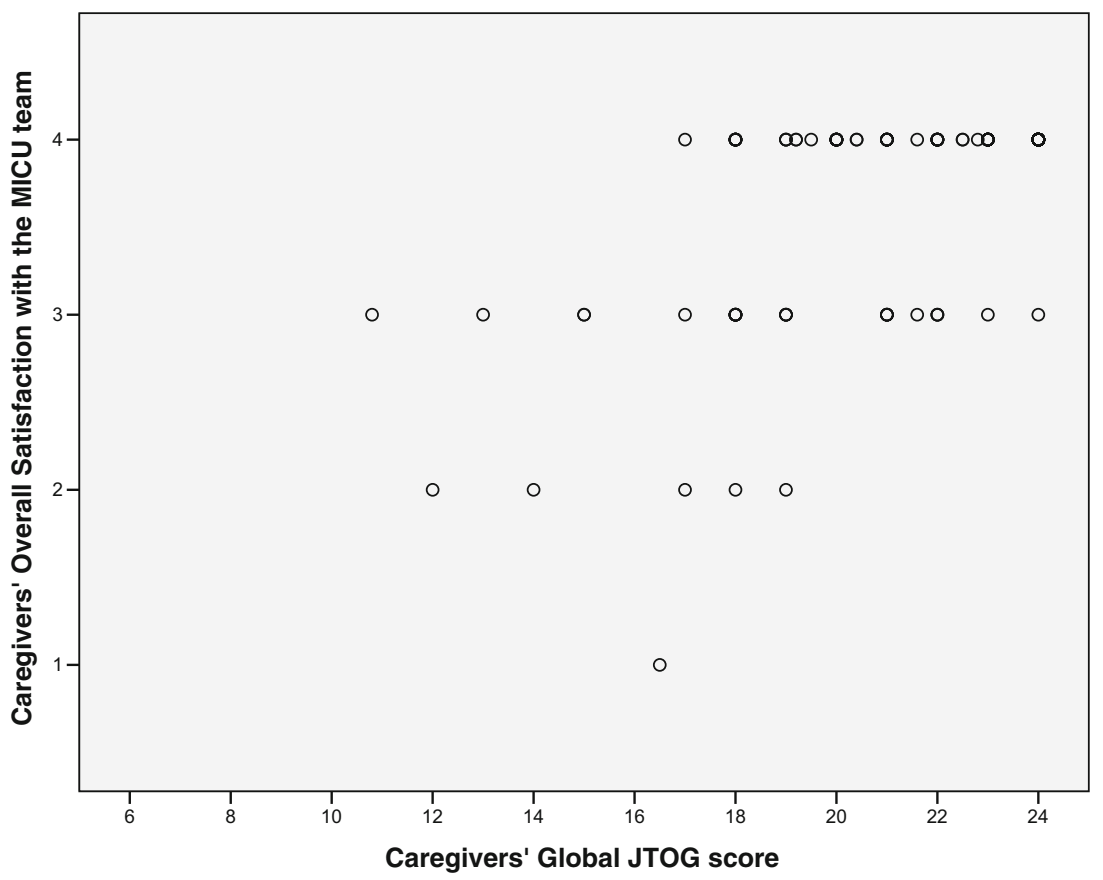

Figure 2 Relationship between caregivers' Global JTOG score (range 6-24) and the single-item question on overall satisfaction with the MICU team (based on Likert scale where 1 is "Not at all satisfied" and 4 is "Extremely satisfied"). Pearson's correlation coefficient, $r=0.596, n=155$, $\mathbf{p}<\mathbf{0 . 0 1}$. 


\section{DISCUSSION}

This study provides unique insight into caregivers' perceptions of collaborative practice behaviors in the MICU and the factors that influence their perceptions. The caregivers surveyed in this study agreed on the importance of IPCP to provide quality healthcare to patients and were satisfied with the care provided by the MICU team. As a group, the caregivers agreed that the MICU team exhibited competency in all four domains of interprofessional collaborative practice. The Support Person JTOG provided reinforcing and constructive feedback on the team's strengths and areas that could be improved in the domains of interprofessional communication and patient/family-centeredness. The JTOG feedback reports positively reinforced the MICU healthcare providers to continue to involve patients and families in daily interprofessional bedside rounds, and the medical residents/students to update family members or support persons about changes in the treatment plan or in patients' clinical status.

The findings in this study point towards the importance of effective communication of information related to the care of MICU patients. Because patients in the MICU may be too ill to participate in decision-making related to treatment and often are unable to provide their perspectives on the functioning of the healthcare team, caregivers are often the key recipients of information and the healthcare proxies. Information communicated to caregivers from healthcare providers creates a framework for understanding a patient's diagnosis, prognosis, and treatment plan; for making informed decisions; and for coping with the psychological stress associated with hospitalization in the MICU. Consistent with our findings, in a descriptive study to understand the needs of family members of ICU patients, Molter found that caregivers want honest information and the assurance that their loved one is being cared for by compassionate hospital staff members. ${ }^{15}$ In a multi-center Canadian study examining family satisfaction with care in the ICU, Heyland et al. demonstrated that overall satisfaction was associated with completeness of information received as well as respect and compassion shown to the patient and family member. ${ }^{16}$ In contrast, Wallau et al. found that miscommunication was the main predictor of caregivers' dissatisfaction with the quality of care provided to patients in a study conducted in an ICU at a university hospital in Brazil. ${ }^{17}$

At our institution, all four versions of the JTOG (Team, Patient, Support Person, and Individual) are utilized in numerous clinical, simulation, and educational settings to assess healthcare providers', students', patients', and caregivers' perceptions of IPCP. Since September 2015, the Team JTOG has been completed by more than 3100 interprofessional learners and clinical providers, the Patient JTOG by over 400 patients, and the Individual JTOG by over 14,000 individuals from practice and educational teams. Practice teams, providers, and learners use the JTOG feedback reports to guide rapid- cycle performance improvement. For example, at our institution, the Patient JTOG has been successfully used to support a new healthcare delivery model for liver transplant evaluation where providers from multiple professions are physically colocated, as well as in a Family Medicine outpatient practice that now incorporates required TeamSTEPPS ${ }^{\circledR}$ communication skills training for all healthcare team members, from medical assistants to attending physicians. ${ }^{18}$ The Support Person JTOG has been used by the inpatient palliative care service after hosting family meetings and led to the reassessment of strategies used by this interprofessional team for breaking bad news. The Individual and Team JTOG are now completed by all learners as part of their required core simulation-based interprofessional education. All participating teams have indicated that the JTOG feedback reports have been useful in supporting efforts at improving IPE and CP initiatives. Faculty can track student participation in IPCP experiences, students are able to track their own Individual JTOG scores over time to assess attainment of competency in IPCP, and patients/families/support persons are able to have their voices heard as part of a comprehensive assessment of IPCP. A 360-degree assessment of practice teams that incorporates the voice of the patient, caregiver, and provider/learner provides a robust assessment of the team's strengths and areas for improvement in team functioning and is valuable in supporting IPCP initiatives.

While the results are promising, there were several limitations of the study design and implementation. First, a small convenience sample from a single academic institution was used, with sample size limited by availability of research assistants to perform data collection. Thus, the perceptions provided by caregivers in this sample are not representative and cannot be generalized to all populations. Second, the study did not obtain any information about the patients whose caregiver was surveyed. Information about patients' admission diagnoses, comorbidities, illness severity, length of stay, and clinical outcomes could shed additional light on caregivers' positive and negative survey responses and perhaps ultimately lead to linkages between effective IPCP and patient outcomes. Theoretically, patients who experience better outcomes or prognoses could potentially influence caregivers to provide positively skewed answers. However, caregivers were asked to provide real-time feedback about a snapshot of their experience with the healthcare team and not feedback based on an entire MICU stay. Further, response bias may be an issue in that caregivers who were more satisfied with care may have been more likely to participate, thus skewing the results in a positive direction. However, the JTOG is not a satisfaction survey, but one focused on evaluation of team behaviors. Fourth, no distinction was made between data collected from caregivers surveyed in the resident- and fellow-staffed MICU and that in the nurse practitioner-staffed MICU. However, the workflow is similar and Scherzer et al. found no significant difference in patient mortality between the two MICUs at our institution. ${ }^{19}$ Despite these limitations, the study provided 
unique insight into caregivers' perspectives on specific team behaviors, which is necessary to inform quality improvement initiatives.

Future studies could use the Support Person JTOG to determine if there is a relationship between specific patient outcomes such as illness severity or length of stay, and caregivers' perceptions of team interactions and competencies for IPCP. If a correlation exists between these two variables, interventions to promote optimal IPCP and teamwork in the clinical setting could be developed with the goal of improving patient care and outcomes. Additionally, the Support Person JTOG could be used to assess the degree of change in caregivers' perceptions of competency in IPCP pre- and postintervention for quality improvement initiatives in both the inpatient and outpatient setting.

\section{CONCLUSIONS}

This study provides valuable information on caregivers' perspectives of the functioning of the interprofessional team in a large, urban academic medical center MICU. Findings underscore the importance of communication and providing patient/ family-centered care by treating patients with respect and by including family members or support persons in discussions about treatment plan.

Acknowledgements: We would like to thank Dr. Marianna LaNoue for her assistance with data analysis. The activities reported here were supported (in part) by the Josiah Macy Jr. Foundation. This project was also supported (in part) by the Health Resources and Services Administration (HRSA) of the U.S. Department of Health and Human Services (HHS) under grant number and title for grant amount (TOBHP28581, Accelerating Primary Care Transformation at Jefferson (JeffAPCT); Total award amount: \$350,000). This information or content and conclusions are those of the authors and should not be construed as the official position or policy of, nor should any endorsements be inferred by HRSA, HHS, or the US Government.

Corresponding Author: Angela M. Gerolamo, PhD, RN; Jefferson College of Nursing, Philadelphia, PA, USA (e-mail: Angela. gerolamo@jefferson.edu).

Compliance with Ethical Standards: This study was exempt from Institutional Review Board review

Conflict of Interest: The authors declare that they do not have a conflict of interest.

Abbreviations

MICU

IPCP

JTOG
IOM

IPE

$\mathrm{CP}$

IPEC
Institute of Medicine

interprofessional education

collaborative practice

Interprofessional Education Collaborative

\section{REFERENCES}

1. Committee on Quality Health Care in America, Institute of Medicine. Crossing the Quality Chasm: A New Health System for the 21st Century. Washington, D.C.: National Academy Press; 2001.

2. Education Committee on Measuring the Impact of Interprofessional. Measuring the Impact of Interprofessional Education on Collaborative Practice and Patient Outcomes. Washington, D.C.: The National Academies Press; 2015.

3. Berwick DM, Nolan TW, and Whittington J. The triple aim: care, health, and cost. Health Aff 2008;27(3):759-769.

4. Institute of Medicine. Measuring the Impact of Interprofessional Education on Collaborative Practice and Patient Outcomes. Washington, D.C.: The National Academies Press; 2015.

5. Reeves S, Fletcher S, Barr H, et al. A BEME Systematic Review of the Effects of Interprofessional Education: BEME Guide No. 39. Med Teach 2016;38(7):656-668.

6. Canadian Interprofessional Health Collaborative. A National Interprofessional Competency Framework. Vancouver, BC: Her Majesty the Queen in Right of Canada; 2010.

7. Davidson JE, Powers K, Hedayat KM, et al. Clinical practice guidelines for support of the family in patient-centered intensive care unit: American College of Critical Care Medicine Task Force 2004-2005. Crit Care Med 2007;35(2):605-622.

8. Burr G. Contextualizing critical care family needs through triangulation: an Australian study. Intensive Crit Care Nurs 1998;14(4):161-169.

9. Al-Mutair AS, Plummer V, O'Brien A, Clerehan R. Family needs and involvement in the intensive care unit: a literature review. J Clin Nurs 2013;22:1805-1817

10. Quinn JR, Schmitt M, Baggs JG, Norton SA, Dombeck MT, Sellers CR. Family members' informal roles in end-of-life decision making in adult intensive care units. Am J Crit Care 2012;21(1):43-51.

11. Lyons KJ, Giordano C, Speakman E, Smith K, Horowitz JA. Jefferson Teamwork Observation Guide (JTOG): an instrument to observe teamwork behaviors. J Allied Health 2016;45(1):49-53.

12. Interprofessional Education Collaborative Expert Panel. Core Competencies for Interprofessional Collaborative Practice: Report of an Expert Panel. Washington, D.C.: Interprofessional Education Collaborative; 2011.

13. Interprofessional Education Collaborative. Core Competencies for Interprofessional Collaborative Practice: 2016 Update. Washington, D.C.: Interprofessional Education Collaborative; 2016.

14. LaNoue M, Collins LG, Sicks S, Asensio A, and Silverio A. Validation study of patient version of the Jefferson Teamwork Observation Guide (JTOGC). J Interprofessional Educ Pract. (Accepted, at press).

15. Molter NC. Needs of relatives of critically ill patients: a descriptive study. Heart Lung 1979;8:332-339.

16. Heyland DK, Rocker GM, Dodek PM, et al. Family satisfaction with care in the intensive care unit: Results of a multiple center study. Crit Care Med 2002;30(7):1413-1418.

17. Wallau RA, Guimaraes HP, dos Reis Falcao LF, et al. Qualidade e Humanização do Atendimento em Medicina Intensiva. Qual a Visão dos Familiares? Rev Bras Ter Intensiva 2006;18(1):45-51.

18. National Center for Interprofessional Practice and Education. Assessing Teamwork: Stories from the Field, A Practical Guide: Volume 4. Regents of the University of Minnesota; 2017.

19. Scherzer R, Dennis M, Swan BA, Kavuru MS, and Oxman D. A Comparison of usage and outcomes between nurse practitioner and resident-staffed medical ICUs. Crit Care Med 2017;45(2):e132-e137. 